\title{
Cholera Outbreak in Yemen: A Regional or a Global Health Crisis?
}

\author{
Farzad Khademi, ${ }^{1}$ Arshid Yousefi-Avarvand, ${ }^{2}$ and Hamid Vaez ${ }^{3,}$ \\ ${ }^{1}$ Department of Microbiology, School of Medicine, Ardabil University of Medical Sciences, Ardabil, Iran \\ ${ }^{2}$ Department of Medical Bacteriology and Virology, School of Medicine, Mashhad University of Medical Sciences, Mashhad, Iran \\ ${ }^{3}$ Department of Microbiology, School of Medicine, Zabol University of Medical Sciences, Zabol, Iran \\ "Corresponding author: Hamid Vaez, PhD, Department of Microbiology, School of Medicine, Zabol University of Medical Sciences, Zabol, Iran. E-mail: hamidvaez@hotmail.com
}

Received 2017 September 30; Revised 2017 December 31; Accepted 2018 March 05.

Keywords: Cholera, Outbreak, Yemen

\section{Dear Editor,}

Vibrio cholerae (V. cholerae) are Gram - negative, curved, rod - shaped and facultatively anaerobic bacteria classified in Vibrionaceae family. These flagellated bacteria naturally grow and survive in contaminated waters around the world and leads to acute diarrheal infection in small intestine of human $(1,2)$. Cholera infection has various symptoms, according to severity of the disease, ranging from self - limited diarrhea to lethal diarrhea along with dehydration and shock. In endemic areas, the disease is transmitted from person - to - person and fecal contamination of drinking water and foodstuff with the bacteria. According to the latest reports, every year, there are 3 to 5 million cases of cholera with 100,000 to 120,000 deaths in the world $(1,2)$. Recently, several cases of $V$. cholera - related epidemic occurred worldwide for various reasons including Bangladesh cholera following the floods (2004), Zimbabwe (2008 to 2009), and Haiti cholera (2010) following the devastating earthquake (1). In the latest cholera epidemic, Yemen faces the most severe form of disease in the world. Yemen is a war - ravaged country on the Arabian Peninsula in the Middle - East region with a population of 27 million. Since the imposition of war by Saudi Arabia and its regional allies against this country in 2015, cholera disease is spreading rapidly with an infection rate of 5000 individual/day (3). According to the latest reports from the World Health Organization (WHO), the number of suspected cholera cases and deaths reached half a million and nearly 2000 individuals, respectively (3). Evidence suggests that during 2 years of civil war more than $40 \%$ of all cases are children under 15 . Cholera affected areas, regions with the prevalence rate $>65 \%$, and morbidity and mortality rate $70 \%$ are shown in Figure 1 based on reports from the Yemen Ministry of Public Health. Disease treatment is possible in several ways including rapid replacement of fluid and electrolytes, and elimination of the organism by antibiotic therapy that leads to reduced toxin production and bacterial transmission $(1,2)$. Furthermore, with the improvements in sanitation conditions through water purification and sewage management as well as nutritional health, the disease can be well controlled and the morbidity and mortality rate decreases from $70 \%$ to less than $1 \%$ in patients. However, inappropriate sanitation conditions such as contaminated drinking water along with malnutrition or contaminated food and prevention of international humanitarian actions due to sea, air, and ground siege as well as the bombing of Yemen have made it difficult to control the disease (4). Despite all problems, the WHO delivered many medical supplies and equipment to Yemen including diagnostic sets, hospital equipment, ambulances, cholera treatment kits, and bags of intravenous fluids (3). Nevertheless, there are not enough treatment centers and medicine. To control the disease and end the crisis of cholera epidemic in Yemen, several attempts are urgently needed including stopping the blockade and bombing of Yemen and use political means to settle the crisis, supporting the health and treatment systems such as restoration of destroyed health and medical infrastructure, access to sufficient healthy water and food, promotion of hygiene and sanitation, releasing humanitarian aids and improving nutrition status, and using oral cholera vaccine (OCV). There are 3 confirmed cholera vaccines to control the outbreak. A large number of laboratories confirmed that cholera outbreak is associated with $V$. cholerae O1. Therefore, employment of these vaccines that are successfully used in various endemic countries confers protection against $V$. cholerae O1. Currently, the outbreak of cholera in Yemen is a regional crisis and if this trend continues, it may turn into a major global problem. If sporadic and epidemic cases of cholera disease are not controlled, worldwide spread of the disease occurs and the world probably faces the 8th cholera pandemic especially in communities with poor sanitation such as Middle - East and African countries $(5,6)$. 


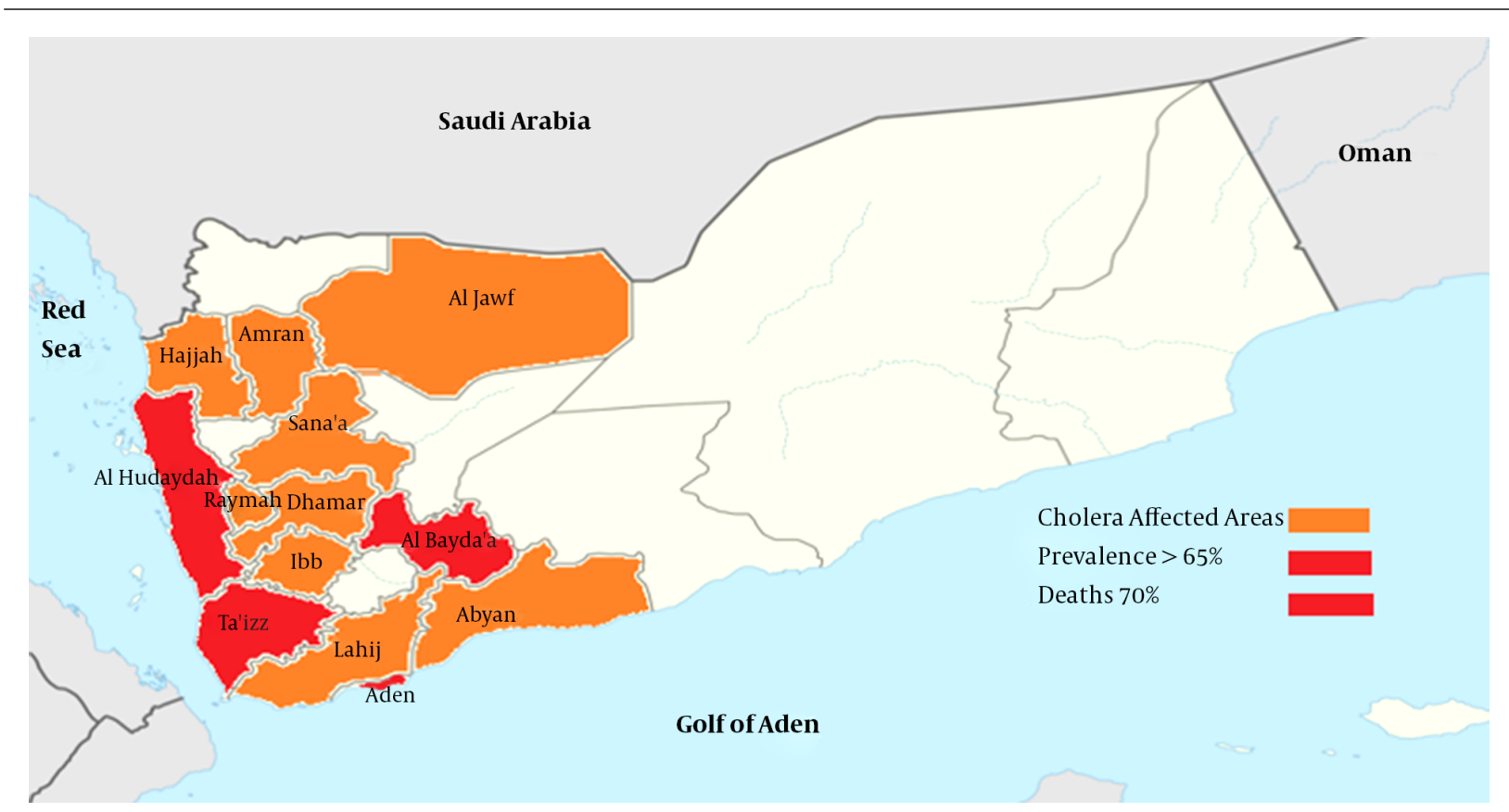

Figure 1. Schematic View of Yemen Cholera Outbreak

\section{References}

1. Murray PR, Rosenthal KS, Pfaller MA. Murray's medical microbiology. 8 th ed ed. USA: Elsevier; 2015

2. Nishiura H, Tsuzuki S, Yuan B, Yamaguchi T, Asai Y. Transmission dynamics of cholera in Yemen, 2017: a real time forecasting. Theor Biol Med Model. 2017;14(1):14. doi: 10.1186/s12976-017-0061-x. [PubMed: 28747188].

3. World Health Organization . Weekly update-Cholera cases in Yemen. 2017. Available from: http://www.who.int/emergencies/yemen/en/.
4. Kupferschmidt K. Cholera vaccine faces major test in Yemen. Science. 2017;356(6345):1316-7. doi: 10.1126/science.356.6345.1316. [PubMed: 28663447].

5. Ali M, Lopez AL, You YA, Kim YE, Sah B, Maskery B, et al. The global burden of cholera. Bull World Health Organ. 2012;90(3):209-218A. doi: 10.2471/BLT.11.093427. [PubMed: 22461716].

6. Shin S, Desai SN, Sah BK, Clemens JD. Oral vaccines against cholera. Clin Infect Dis. 2011;52(11):1343-9. doi: 10.1093/cid/cir141. [PubMed: 21498389]. 